\section{Random Fields}

Ibrahim Ben Daya

Albert I.H. Chen

Mohammad Javad Shafiee

John T.W. Yeow

Alexander Wong
University of Waterloo, ON, Canada

University of Waterloo, ON, Canada

University of Waterloo, ON, Canada

University of Waterloo, ON, Canada

University of Waterloo, ON, Canada

\section{Abstract}

The row-column method is a simplification technique used to reduce the complexity of a fully addressed 2-D array. Although it greatly reduces the number of physical connections required as well as the amount of data to be handled, it still has limitations; its imaging data output is sparse, it suffers from speckle noise, and its spatially-dependant point spread function is riddled with edge artifacts. In this work, we propose a row-column ultrasound imaging system, termed CRUIS3D, that uses a 3-D edge-guided random field approach to compensate for the limitations of the row-column method. Tests on CRUIS3D and previously published row-column systems show the effectiveness of our proposed system as a tool for enhancing 3-D row-column ultrasound imaging.

\section{Introduction}

3-D ultrasound imaging offers opportunities that cannot be found in 2-D imaging [1]. To acquire a 3-D ultrasound image, either a 1-D array is mechanically moved to cover a volume (which introduces unwanted artifacts) or a 2-D fixed transducer is used. Although a 2-D transducer is preferred, a fully addressed $N \times N$ array requires $N^{2}$ connections. This poses a challenge both in physically addressing the high number of connections as well as dealing with large amount of data produced [1]. One way to simplify the fully addressed 2-D array is to use an orthogonal set of $N$ rows and $N$ columns, as shown in figure 1 , where one set could be responsible for transmit beamforming and the other for receive beamforming. Using this row-column method, which was first proposed in [2], the number of connections required is reduced to $N+N$.

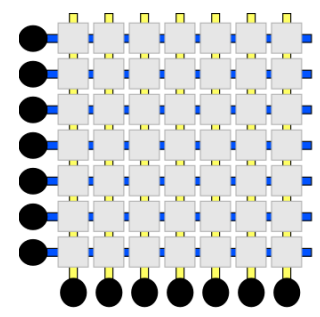

a)

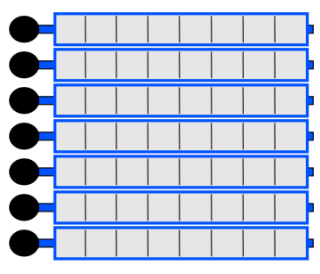

b)

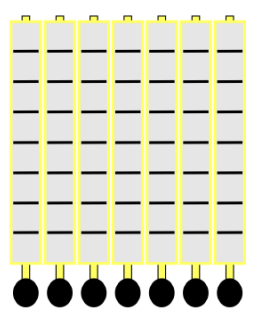

C) edge-guided stoachastically fully connected random fields that is capable of addressing the row-column method's limitations.

The rest of the document is organized as follows: Section 2 will outline the methodology our proposed system follows, Section 3 will detail the tests we performed and discuss the results, Section 4 concludes the document.

\section{Method}

The proposed system can be outlined in two parts: characterization and compensation.

\subsection{Characterization}

Three models are used to characterize the baseline RC system: an image formation model, a noise model, and a PSF model. All three will now be outlined.

\subsubsection{Image formation model}

Image formation can be described using:

$$
g_{r}(x, y, z)=M(x, y, z)[f(x, y, z) * h(x, y, z)+u(x, y, z)] .
$$

where $x, y$, and $z$ are the Cartesian coordinates. The term $g_{r}(x, y, z)$ is the observed RF image, $M(x, y, z)$ is the data acquisition unit's sampling function, $f(x, y, z)$ represents the tissue reflectively function, the operator ' $*$ ' denotes the convolution operation, $h(x, y, z)$ represents the PSF function (PSF), and $u(x, y, z)$ is the noise component.

\subsubsection{Noise model}

In [7], a number of distributions to model speckle were proposed. Empirical testing done on our captured data showed that the best fit is the Fisher-Tippett distribution:

$$
p(I(x, y, z))=2 \exp \left[\left(2 I(x, y, z)-\ln 2 \sigma^{2}\right)-\exp \left[2 I(x, y, z)-\ln 2 \sigma^{2}\right]\right]
$$

where $I$ is voxel intensity and $\sigma$ is the standard deviation.

Fig. 1: The row-column setup a) is a combination of two orthogonal sets of 1-D arrays arranged in rows b) and columns $c$ )

The row column method still suffers from a few intrinsic limitations: data is inherently sparse, it suffers from speckle noise found in all ultrasound images, and the point spread function (PSF) is spatially dependant and suffers from edge artifacts. A few proposed row-column ultrasound imaging systems attempt to address some of these limitations. Some attempted to focus primarily on transducer design to improve image quality. These include a system proposed by [3], which we will use as a baseline system and will henceforth be referred to as baseline RC, and a system that integrates apodization in its transducer design, presented in [4] and [5]. Another group of proposed systems attempt to address the row-columns limitations algorithmically. In [1], a compensated system was proposed that uses a multilayared conditional random field (MCRF) based approach to address these limitations, another [6] was proposed with an extended MCRF that leverages information in 3-D to get better images. Both systems had a problem with edge preservation and tended to oversmooth images. In this research, we propose CRUIS3D a compensated row-column ultrasound imaging system that leverages information in 3-D using an

\subsubsection{Point spread function model}

Sound pressure emitted from the transducer weakens as it moves away from the transducer [8], creating a varying beam profile that needs to be taken into account in order to compensate for it. For the spatially dependant PSF of the baseline RC system, we use a model based on the Tupholme-Stephanisshen model for spatial impulse response that was furthur derived for the pulse echo case in [9]. According to this model, the one way impulse response of a row-column system at point $\vec{r}_{1}$ with a transducer of geometry $S$ and point $\overrightarrow{r_{2}}$ is is given by:

$$
h\left(\overrightarrow{r_{1}}, \overrightarrow{r_{2}}, t\right)=\int_{S} \frac{\delta\left(t-\frac{\left|\overrightarrow{r_{1}}-\overrightarrow{r_{2}}\right|}{c}\right)}{2 \pi\left|\overrightarrow{r_{1}}-\overrightarrow{r_{2}}\right|} \mathrm{d} S
$$

$c$ here is the speed of sound at a homogeneous medium and $\delta($. is the Dirac delta function. The PSF can then be expressed as:

$$
H_{p e}\left(\overrightarrow{r_{1}}, \overrightarrow{r_{2}}, t\right)=h\left(\overrightarrow{r_{1}}, \overrightarrow{r_{2}}, t\right) * h\left(\overrightarrow{r_{2}}, \overrightarrow{r_{1}}, t\right)
$$

These three models are used to characterize the three intrinsic limitations mentioned earlier. We will use them to compensate for these limitations in the second stage. 


\subsection{Compensation}

The compensation stage attempts to solve the inverse problem of equation 1, leveraging information from the characterization stage. Is is essentially an image reconstruction problem, and to solve it we formulate it as a Maximum a Posteriori (MAP) problem, where a posterior distribution $P(F \mid G)$ is maximized:

$$
F^{*}=\underset{\bar{F}}{\operatorname{argmax}}\{P(F \mid G)\}
$$

where $F^{*}$ is the MAP solution, $\bar{F}$ is the possible results set, and $G$ is the observation.

To model this conditional probability without specifying a prior model, we can use conditional random fields (CRFs). First proposed by [10], CRFs are generally expressed in the form:

$$
P(F \mid G)=\frac{1}{Z(G)} \exp (-\psi(F, G))
$$

with $Z$ being the partition function and $\psi(\cdot)$ a combination of any arbitrary unary and pairwise potential functions:

$$
\psi(F, G)=\sum_{i=1}^{n} \psi_{u}\left(f_{i}, G\right)+\sum_{c \in C} \psi_{p}\left(f_{c}, G\right)
$$

with $C$ being a set of a clique structure for each node.

The general CRF model considers local interactions within relatively small clique structures, while fully connected conditional random fields (FCRFs) consider node interaction within a global scale [11]. FCRFs has a huge computational cost, one way to address this cost is to consider stochastically fully connected random fields (SFCRF), where a clique structure is based on various indicator functions. A clique structure $C$ can be represented as:

$$
\begin{gathered}
C=\left\{C_{p}(i)\right\}_{i=1}^{n} \\
C_{p}(i)=\left\{(i, j) \mid j \in N(i), 1_{\{i, j\}}^{S}=1\right\}
\end{gathered}
$$

with the stochastic indicator function $1_{\{i, j\}}^{S}$ defining whether two nodes can construct a clique. The function used in the proposed system is a combination of three probability distributions: one based on spatial information $P_{i, j}^{s}$, another based on data relation among states $Q_{i, j}^{d}$, and the final one based on edge information $R_{i, j}^{e}$. The full stochastic indicator function is expressed as:

$$
1_{\{i, j\}}^{S}= \begin{cases}1 & P_{i, j}^{s} \cdot Q_{i, j}^{d} \cdot R_{i, j}^{e} \geq \gamma \\ 0 & \text { otherwise }\end{cases}
$$

With the clique structure set by this indicator function, we can now discuss the potential functions.

The unary potential function is a data driven function that incorporates the information corresponding to the observation into the random field model. It also incorporates the spatially varying PSF, and is formulated according to the noise model presented in equation 2 , since that is how we assume the image is degraded.

The pairwise potential function incorporates spatial information into the model. These functions act on the subset of random variables in the clique structure set by the stochastic indicator function from equation 10. The pairwise function used in this system is based on two penalty terms:

- Spatial proximity penalty: this penalizes voxels that are far away since we assume that the farther away a voxel is, the less likely it belongs to the same label

- First order variation penalty: this helps preserve the boundaries of the estimated image by using differences in intensity to outline tissue transitions [1].

With the potential functions defined, we can formulate an energy function to drive the MAP problem:

$$
E(F, G, C r)=\sum_{i=1}^{n} \psi_{u}\left(f_{i}, G, C r_{i}\right)+\sum_{c \in C} \psi_{p}\left(f_{c}, G\right) .
$$

Equation 5 can be reformulated as:

$$
F^{*}=\underset{\bar{F}}{\operatorname{argmin}}\{E(F, G, C r)\} .
$$

\begin{tabular}{llll}
\hline System & PSNR (dB) & CoC & ENL \\
\hline CRUIS3D & $\mathbf{3 1 . 9 2 5 4}$ & $\mathbf{0 . 3 6 2 2}$ & 0.3062 \\
3D-CRC [6] & 30.2045 & 0.2050 & $\mathbf{0 . 4 8 5 1}$ \\
Baseline RC [3] & 27.3844 & 0.0198 & 0.2355 \\
Integrated Apodization [4] & 26.2299 & 0.0201 & 0.1618 \\
Fully addressed 2-D & 27.9111 & 0.0078 & 0.0371 \\
\hline
\end{tabular}

Table 1: Quantitative evaluation for the simulated phantom. The proposed CRUIS3D outperforms all systems in literature when it comes to PSNR and CoC, while the oversmoothing 3D-CRC has higher ENL.

Gradient descent algorithm is used to solve this MAP problem.

With data sparsity taken into account through the pairwise potential function, and speckle noise as well as the spatially dependant PSF taken into account, all the suggested intrinsic limitations are now addressed. The edge guided stochastic indicator function along with the longer range internodal connection of the SFCRF model should strengthen and maintain inhomogenous areas and preserve edges by preventing oversmoothing. We will now test our proposed system.

\section{Results}

In order to evaluate our proposed system, we used simulated ultrasound scans and compared our system against previously published systems in literature to highlight the value of incorporating the edge guided approach and leveraging information in 3-D.

\subsection{Simulation}

Ultrasound imaging simulation was done using the open source MATLAB toolkit Field II [12]. Beamfoming in a manner similar to the row-column method was performed. Envelope data from the scans were mapped using linear interpolation into a 3-D lattice and passed to the compensation stage.

The phantom being tested consists of four point source in 20 $\mathrm{mm} \times 20 \mathrm{~mm} \times 60 \mathrm{~mm}$ volume. The point source are located at $[x, y, z]=(0,0,30),(0,0,35),(2,2,40)$, and $(-2,-2,40) \mathrm{mm}$.

\subsection{Quantitative Evaluation}

Peak signal-to-noise ration (PSNR), coefficient of correlation (CoC), and expected number of looks (ENL) are the metrics used to evaluate the performance of our system. All three metrics were defined according to recent literature[13, 14]. Quantitative comparison between CRUIS3D and other systems in literature are summarized in table 1.

Quantitative analysis shows that CRUIS3D outperforms 3DCRC in both PSNR and CoC, indicating better noise suprresion as well as edge preservation. 3D-CRC had a higher ENL value indicating smoother regions, meaning CRUIS3D edge guided approach did address the oversmoothing issue 3D-CRC has. CRUIS3D outperformed all other systems across the three metrics.

\subsection{Visual Evaluation}

A 2-D slice taken from the middle of the 3-D outputs of all systems is shown in figure 2. A closer look at the reconstruction of each point source is shown in figure 3 The top point source was best reconstructed with CRUIS3D and 3D-CRC, while the middle point source is best seen in the fully addressed approach. The bottom two point sources (which should not be seen in this middle slice) are mostly suppressed in all systems but is clearly visible in the fully addressed array. CRUIS3D suppressed the ringing artifacts seen in the baseline RC and the top and bottom points source in the fully addressed array, while having a closer look to a point source than the integrated apodization system. 3D-CRC has bigger point sources than CRUIS3D, showing our edge guided approach addressed the oversmoothing issue. These observations are supported by the quantitative evaluation. 

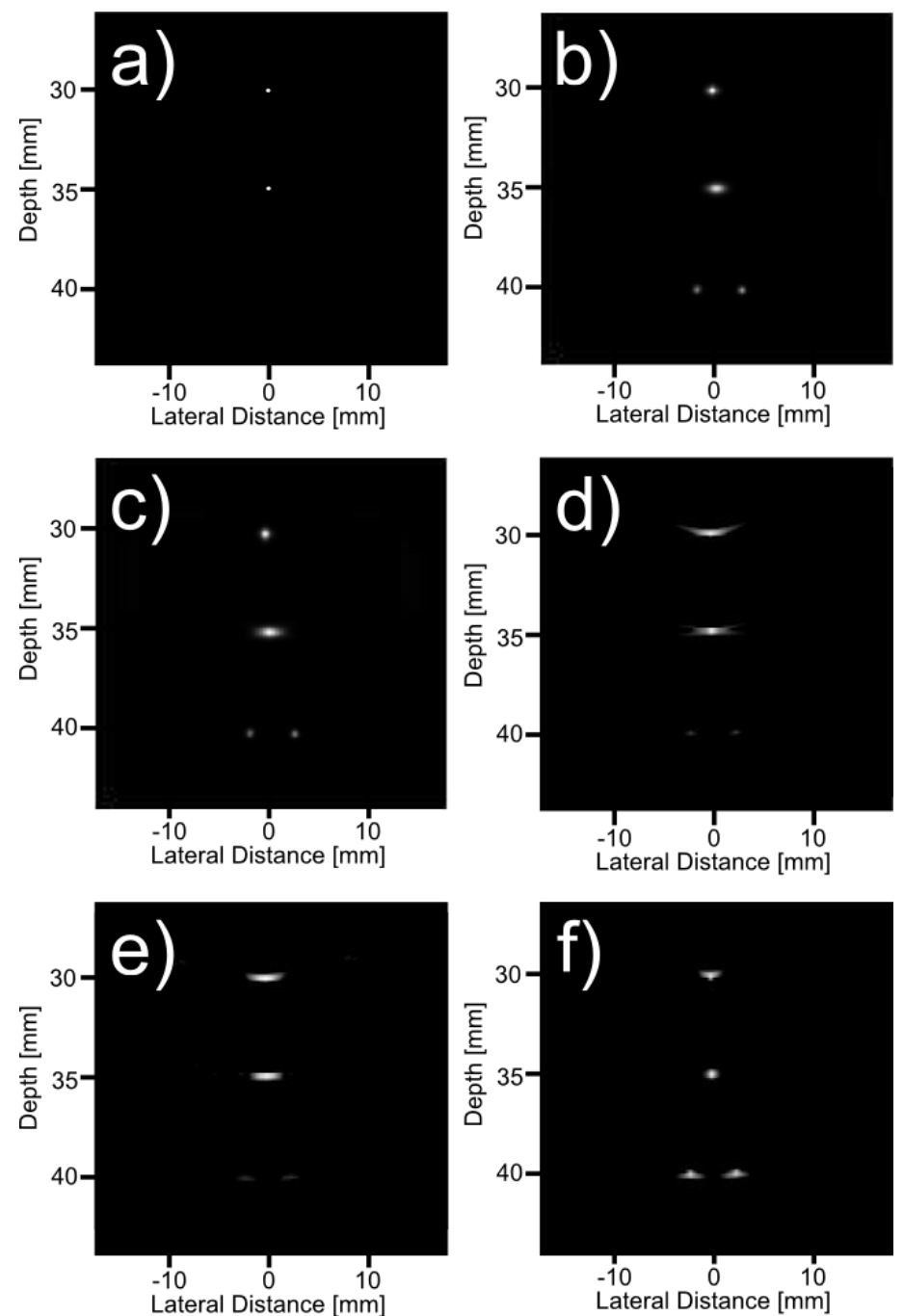

Fig. 2: Visual comparison between the proposed system and other systems in literature. a) shows the phantom, b) is the image from CRUIS3D, c) is image from 3D-CRC[6], d) is the image from the baseline $\mathrm{RC}$ [3], e) is the image from integrated apodization system [4], and f) is the image from a fully addressed 2-D array.

\section{Conclusion}

In this work, we proposed CRUIS3D: a compensated row-column ultrasound imaging system that leverages $3-D$ information within a stochastically fully connected 3-D random field with an edge guided stochastic indicator function that is capable of addressing the intrinsic limitations of the row-column method. We achieved state of the art performance compared against other published row-column systems in literature under PSNR, CoC, and ENL as well as through visual evaluation. This shows the value of edge-preservation to prevent oversmoothing.

\section{Acknowledgments}

This research was funded by the Natural Sciences and Engineering Research Council of Canada as well as the Canada Research Chairs Program.

\section{References}

[1] Ben Daya, I., Chen, A. I. H., Shafiee, M. J., Wong, A., and Yeow, J. T. W. Compensated row-column ultrasound imaging system using fisher tippett multilayered conditional random field model. PLoS ONE 10(12), e0142817, Dec (2015).

[2] Morton, C. and Lockwood, G. Theoretical assessment of a crossed electrode 2-d array for 3-d imaging. IEEE Symposium on Ultrasonics 1, 968 - 971, (2003).

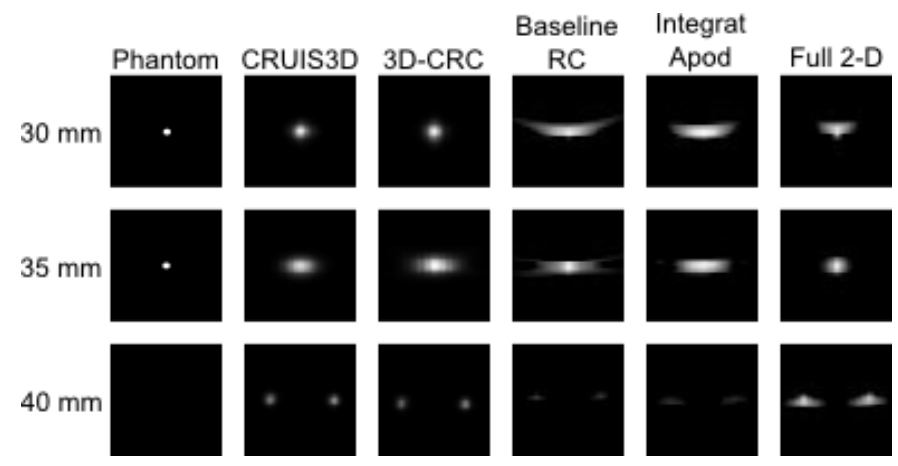

Fig. 3: A closer look at the reconstruction of point sources

[3] Chen, A., Wong, L., Logan, A., and Yeow, J. T. W. A cmutbased real-time volumetric ultrasound imaging system with row-column addressing. IEEE International Ultrasonics Symposium, 1, 1755 - 1758, (2011).

[4] Rasmussen, M., Christiansen, T., Thomsem, E., and Jensen, J. 3-d imaging using row-column-addressed arrays with integrated apodization - part i: apodization design and line element beamforming. IEEE Transactions on Ultrasonics, Ferroelectrics, and Frequency Control 62(5), 947 - 958, (2015).

[5] Christiansen, T. et al. 3-d imaging using row-columnaddressed arrays with integrated apodization - part ii: transducer fabrication and experimental results. IEEE Transactions on Ultrasonics, Ferroelectrics, and Frequency Control 62(5), 959 - 971, May (2015).

[6] Ben Daya, I., Chen, A. I. H., Shafiee, M. J., Wong, A., and Yeow, J. T. W. Compensated Row-Column Ultrasound Imaging System Using Three Dimensional Random Fields Image Analysis and Recognition: 14th International Conference, 107-116, (2017).

[7] Michailovich, O. and Tannenbaum, A. Despeckling of medical ultrasound images. IEEE Transactions on Ultrasonics, Ferroelectrics, and Frequency Control 53(1), 64 - 78, Jan (2006).

[8] Szabo, T. L. Diagnostic ultrasound imaging: inside out. Elsevier Academic Press (2004).

[9] Jensen, J. A model for the propagation and scattering of ultrasound in tissue J.Acoust.Soc.Am. 89(1), pp. 182-191, Jan (1991).

[10] Lafferty, J. D., McCallum, A., and Pereira, F. C. N. Conditional random fields: probabilistic models for segmenting and labeling sequence data. Proceedings of the Eighteenth International Conference on Machine Learning, 282 - 289 (2001).

[11] Shafiee, M. J., Wong, A., Siva, P., and Fieguth, P. Efficient bayesian inference using fully connected conditional random fields with stochastic cliques. IEEE Intrnational Conference on Image Processing, 4289 - 4293 (2014).

[12] Jensen, J., A. Field: a program for simulating ultrasound systems. 10th Nordic-Baltic Conference on Biomedical Imaging Published in Medical Biological Engineering Computing 34, 351 - 353, (1996).

[13] Wu, S., Zhu, Q., and Xie, Y. Evaluation of various speckle reduction filters on medical ultrasound images. Engineering in Medicine and Biology Society, 1148 - 1151, July (2013).

[14] Nageswari, C. and Prabha, K. Despeckle process in ultrasound fetal image using hybrid spatial filters. International Conference on Green Computing, Communication and Conservation of Energy, 174 - 179, Dec (2013). 